Meta

Journal des tradlucteurs

Translators' Journal

\title{
Le cabinet de traduction : Une gestion de l'évolution
}

\section{Raymond Frenette}

Volume 29, numéro 4, décembre 1984

URI : https://id.erudit.org/iderudit/002775ar

DOI : https://doi.org/10.7202/002775ar

Aller au sommaire du numéro

Éditeur(s)

Les Presses de l'Université de Montréal

ISSN

0026-0452 (imprimé)

1492-1421 (numérique)

Découvrir la revue

Citer cet article

Frenette, R. (1984). Le cabinet de traduction : Une gestion de l'évolution. Meta, 29(4), 352-358. https://doi.org/10.7202/002775ar d'utilisation que vous pouvez consulter en ligne.

https://apropos.erudit.org/fr/usagers/politique-dutilisation/ 


\section{LE CABINET DE TRADUCTION : UNE GESTION DE L'ÉVOLUTION}

RAYMOND FRENETTE

\section{INTRODUCTION}

Les cabinets de traduction existent depuis longtemps. Toutefois, à l'exception de certains cabinets ayant obtenu d'importants contrats gouvernementaux, les cabinets de traduction bien structurés, comprenant au moins cinq professionnels de la traduction appuyés par une intendance solide et des ressources techniques appropriées, ont vraiment commencé à voir le jour il y a une dizaine d'années à peine. Auparavant, il s'agissait, la plupart du temps, d'un regroupement de deux ou trois personnes tout au plus, la presque-totalité des besoins en traduction commerciale étant comblée par les services de traduction internes des grandes entreprises. La percée de cabinets de traduction de plus grande importance est due à l'évolution de la situation linguistique au pays et sans doute aussi à certains esprits aventuriers qui ont voulu avoir pignon sur rue, comme bien d'autres professionnels et, les circonstances étant favorables, pourquoi pas? Étant donné donc, l'émergence relativement récente du cabinet de traduction au sens précisé plus haut, il ne faudra pas se surprendre si le texte qui suit véhicule beaucoup plus d'interrogations que d'affirmations ou de conclusions sur la gestion de la traduction. En pleine crise d'identité et en pleine mutation, les cabinets cherchent à se structurer, à établir des normes et à tirer des conclusions. Ce n'est pas facile, mais c'est passionnant, et surtout, c'est davantage possible et réalisable au Québec et au Canada plus que partout ailleurs dans le monde, bien qu'il y ait plusieurs pierres d'achoppement, pour ne pas dire des pièges.

\section{UNE DÉFINITION}

On a dit tant de choses sur le cabinet de traduction et, il faut bien l'avouer, certains cabinets de traduction plus ou moins dignes de ce nom se sont réellement écartés de leur mission fondamentale, qu'il est probablement devenu utile de redéfinir simplement sa nature, sa raison d'être, ses objectifs et ses moyens d'action.

Un cabinet de traduction, c'est un regroupement de traducteurs et de terminologues professionnels d'expérience qui ont décidé de mettre en commun leurs connaissances diverses de même que les moyens financiers et techniques essentiels à l'exercice efficace et rentable de la profession, dans le but d'offrir à une clientèle variée, de la traduction de qualité, peu importe le volume, et ce, à un prix raisonnable, grâce à cette mise en commun de toutes les ressources.

\section{LES RESSOURCES HUMAINES}

Le cabinet de traduction se doit d'abord d'assurer une pratique conforme aux normes et à l'éthique de la profession. Les ressources humaines sont essentielles dans un cabinet de traduction, ce qui ne diffère guère, d'ailleurs, de tout cabinet offrant des services professionnels. Étant donné la nature du produit, les outils et les moyens techniques les 
plus sophistiqués ne sauraient remplacer, du moins dans un avenir prévisible, une équipe de professionnels chevronnés, qu'il s'agisse de traducteurs, de terminologues, de rédacteurs ou de communicateurs.

Il me semble donc essentiel que les associés d'un cabinet de traduction aient une formation appropriée, qu'ils soient membres d'une association professionnelle reconnue (STQ par exemple) et qu'ils comptent au moins cinq ans d'exercice de la profession sous la surveillance de professionnels de la traduction.

Jusqu'à maintenant, les cabinets de traduction n'ont guère joué un rôle de formation auprès des jeunes traducteurs. En effet, la nouveauté relative du cabinet de traduction et les structures à inventer et à instaurer ont accaparé le peu de moments libres des membres du cabinet, les empêchant par le fait même d'accorder à des stagiaires le temps et l'attention voulus pour les aider à progresser. C'est pourquoi, jusqu'à tout récemment, seuls des traducteurs chevronnés avaient leur place au sein d'un cabinet de traduction.

Les choses sont toutefois en train de changer au fur et à mesure que les cabinets de traduction établissent des structures d'accueil favorables à l'épanouissement professionnel des débutants. À ce chapitre, il faut tout de même ajouter qu'il y a autant de différence entre un traducteur qui travaille au sein d'un cabinet et celui qui travaille dans une entreprise, que dans le cas d'un avocat, d'un ingénieur ou d'un comptable dans des situations comparables. Dans le cabinet, les clients sont multiples, tout comme les exigences et les spécialités. D’autre part, le seuil de rentabilité ne peut être négligé sous peine d'entraîner le cabinet dans la faillite. Pour toutes ces raisons, le traducteur qui travaille dans un cabinet, même s'il s'agit d'un traducteur débutant, doit posséder de nettes aptitudes à la polyvalence, il doit rapidement acquérir le sens de la discipline et des méthodes sûres d'évaluation de l'efficacité du temps utilisé, et enfin, une grande capacité à apprendre et à assimiler, afin d'atteindre rapidement une certaine autonomie. En fait, pourquoi les traducteurs débutants seraient-ils traités autrement que les débutants et les stagiaires des autres professions. $\grave{A}$ un débutant, on confie peu de responsabilités et des travaux moins complexes, certes, mais il doit, dès le début, assumer l'entière responsabilité de ce qui lui est confié. Autrement, on établit en principe la révision et la correction systématiques, ce qui, sous réserve de certains cas particuliers, est une aberration qui prive le traducteur de l'autonomie essentielle à l'exercice normal de sa profession et risque d'endormir sa conscience professionnelle.

La composition du cabinet et le nombre de personnes s'établissent nécessairement en fonction des besoins de la clientèle à servir, des secteurs du marché visés et des services que l'on désire offrir. Ceci dit, un cabinet de traduction peut compter autant de traducteurs, de terminologues, de rédacteurs, de communicateurs, de documentalistes, de gestionnaires, d'opérateurs que nécessaire, jusqu'à concurrence d'une vingtaine de personnes. En effet, au-delà de 20 personnes, il devient difficile d'éviter l'allure d'usine et de production de masse, qui, on a pu le constater, tend à affecter le rendement intellectuel et l'engagement personnel unique que requiert la nature du travail. Rien n'empêche, cependant, de créer un ou plusieurs autres groupes de travail électroniquement reliés au premier et partageant également avec lui toutes les ressources qui ont avantage à être mises en commun et qui peuvent l'être.

Il faut également recourir à des ressources humaines extérieures au cabinet, à titre permanent ou temporaire. Par exemple, des traducteurs ou des terminologues engagés sur une base contractuelle ou autre et des spécialistes sans lesquels on mettrait en danger, à long terme, la survie du cabinet de traduction, dont la gestion devient chaque jour plus complexe : ce sont les comptables, les fiscalistes, les conseillers en gestion, les con- 
seillers en marketing et dans certains cas, les conseillers en bureautique et en informatique.

Mais le véritable tour de force consiste à créer une ambiance favorable au travail en collaboration. En effet, toutes ces personnes sont nécessairement autonomes et dynamiques, elles recherchent la satisfaction professionnelle et l'épanouissement dans l'accomplissement de leur travail et ce sont généralement de fortes personnalités, ce qui est parfaitement normal, vu les exigences et les conditions particulières d'exercice de la profession dans un cabinet. L'un des secrets de la réussite de ce travail d'équipe, c'est sans aucun doute la connaissance de soi, de ses qualités, de ses défauts, de ses limites et la capacité de les reconnaître et de les accepter. Se connaître suffisamment pour accepter de contribuer aux efforts de l'équipe selon deux critères : ses aptitudes et ses talents réels, d'une part, et les besoins véritables de l'équipe face à la clientèle, d'autre part.

\section{LES RESSOURCES TECHNIQUES}

Grâce aux ressources techniques, la traduction dans un cabinet n'est déjà plus ce qu'elle était il y a cinq ans, mais ce n'est pas tout, car les prochains mois apporteront probablement encore plus de changements qu'il n'y en avait eu au cours des cinq dernières années. On assiste à une poussée formidable et irrésistible du traitement de textes, de l'informatique et de la télématique.

\section{A. LE TRAITEMENT DE TEXTES}

Voilà un outil puissant, bien qu'encore passablement dispendieux, mais indéniablement rentable, lorsque utilisé à bon escient. Il ne doit pas servir à encourager les traducteurs à " pondre ", à toute vitesse, des textes mal tournés, sous prétexte que les corrections sont rapides et faciles grâce au traitement de textes. En réalité, les interventions successives à l'écran peuvent finir par doubler, sinon tripler le coût d'une frappe normale, et il serait certes inexcusable d'en faire supporter les frais par les clients.

Par contre, si, en plus des opérateurs, les traducteurs doivent eux-mêmes travailler à l'écran, il vaut mieux qu'ils le fassent correctement dès le début, après avoir reçu une formation appropriée, ceci afin d'éviter que l'on gaspille inconsidérément le temps du traducteur et celui de l'utilisation d'une machine dispendieuse qui pourrait certes être utilisée de façon plus efficace et plus rentable par un opérateur bien formé.

Mais ceci étant dit, comment donc avons-nous pu travailler si longtemps sans cet outil précieux devenu indispensable ? Les corrections inévitables se font en vitesse et impeccablement, les relectures fastidieuses sont évitées, le repérage des termes à modifier devient un jeu d'enfant, les possibilités de mise en page et de présentation sont nombreuses et variées, le classement par ordre alphabétique est cent fois plus rapide et enfin, l'acheminement vers l'impression est plus efficace, moins long et surtout, il y a beaucoup moins de risques d'erreurs regrettables ou de coquilles irritantes dans un texte que, par conscience professionnelle, l'on veut toujours parfait et impeccable.

\section{B. LA COMPOSITION}

La machine à photocomposition est un atout si la clientèle le justifie. Il y a toutefois un risque de dispersion des énergies vers un domaine qui est tout de même très secondaire par rapport à l'activité principale du cabinet.

\section{L'ABONNEMENT À UNE BANQUE DE TERMINOLOGIE CENTRALISÉE}

C'est la voie de l'avenir et ce sera bientôt un outil indispensable, à condition toutefois qu'il y ait un assouplissement de la centralisation pour permettre un échange entre la banque centrale et les producteurs de terminologies que sont, entre autres, les cabi- 
nets de traduction. Il faudrait alors procéder à l'informatisation du stock terminologique appartenant au cabinet.

\section{LA DOCUMENTATION}

Une bonne bibliothèque et un fichier terminologique informatisé sont des éléments vitaux pour un cabinet. Toutefois, un cabinet ne saurait acquérir tous les ouvrages de références spécialisés dans tous ses domaines d'exercice ni élaborer toutes les terminologies utiles. En conséquence, il faut éviter le dédoublement des bibliothèques spécialisées déjà bien garnies et des fichiers terminologiques qui sont accessibles, soit dans les institutions d'enseignement, soit dans les entreprises ou les organismes privés ou publics.

\section{E. LES TÉLÉCOMMUNICATIONS}

J'aurais pu parler de télématique, mais pour le moment, je me contenterai de souligner que la communication d'une machine de traitement de textes à une autre est une ressource technique accessible et déjà facilement intégrable. Quelle perspective intéressante que celle qui consiste à relier des traducteurs de cette façon, évitant ainsi les déplacements inutiles, permettant l'interrogation terminologique, sans oublier les économies considérables de temps, de transport, de vêtements et de repas au restaurant, sans parler du plaisir et du confort qui s'ajoutent au fait de travailler à domicile, surtout durant ces longs hivers québécois où les déplacements peuvent devenir si pénibles et difficiles. Quelle incroyable facilité également, que de communiquer directement à un client, en quelques secondes, les quelque cent pages que l'on a traduites, sans risque d'erreurs et sans avoir recours au service de messagerie, peu importe la distance ou la situation géographique.

\section{F. LA TRADUCTION ASSIGNÉE PAR ORDINATEUR}

Certaines expériences sont en cours avec plus ou moins de succès. Il est clair que l'avenir va dans ce sens. Toutefois, il faut être lucide et considérer ces premiers efforts pour ce qu'ils sont : " des expériences de laboratoire " coûteuses qui ne seront rentables, efficaces et utiles pour la clientèle que dans une bonne décennie à tout le moins, sauf dans de rares domaines où les besoins sont précis et requièrent peu de vocabulaire ou de terminologie. Ce sont des efforts prématurés qui ne pourront aboutir qu'au moment où on aura réussi à informatiser et à rendre accessible la terminologie existante. Je ne m'étendrai pas sur la traduction automatique, laquelle relève plutôt de la futurologie, sinon de la science-fiction pour le moment, quoi qu'en disent les informaticiens ou leurs représentants.

\section{UNE GESTION DE L'ÉVOLUTION}

On conçoit facilement, à la lumière de tout ce qui précède, que la gestion d'un cabinet de traduction n'est pas une sinécure. Doit-on acheter du nouvel équipement ? La demande de la clientèle le justifie-t-elle? Faut-il acheter plusieurs systèmes de traitement de textes, étant donné que les clients utilisent divers systèmes qui sont tous incompatibles les uns avec les autres ? Et la recherche terminologique, ponctuelle ou thématique, est-ce bien rentable et qui va payer la note? Et la qualité, sauf nous, les professionnels de la traduction, qui y croit vraiment? Et d'abord qu'est-ce que la qualité, et le client en veut-il ? Nos prix sont-ils vraiment concurrentiels ? Faut-il viser surtout le grand volume, quitte à faire plus vite et un peu moins soigné, ou faut-il maintenir une qualité uniforme en tout ? Le marché québécois est-il unique ou peut-on aborder de la même façon le marché de la traduction à Toronto, Winnipeg, Calgary, Vancouver, Fredericton et Moncton, voire même New York et Los Angeles? Et les gouvernements 
provinciaux et fédéral ... leur offrir nos services, et si oui, à quel prix, et est-il vrai qu'en raison de leur importance, ils contrôlent le marché ? Quelle sera l'évolution du marché d'ici cinq ans ou dix ans ? Que faut-il prévoir tout de suite pour ne pas aboutir à un culde-sac et être acculé à la faillite ? Et l'économie est-elle en reprise réelle ou fictive, et en conséquence, est-il temps de prendre de l'expansion ou, au contraire, de faire preuve de prudence et de restreindre les dépenses, quitte à refuser une clientèle qui requiert l'achat d'un nouvel équipement?

En fait je n'ai pas voulu effrayer ni décourager personne en posant toutes ces questions, mais seulement faire ressortir à quel point les traducteurs peuvent être aussi mal armés pour assurer la gestion d'un cabinei de traduction que tout entrepreneur qui se lance à son compte sans avoir acquis des notions de gestion des ressources humaines et financières. La question est importante, car il y a beaucoup de similitudes entre la gestion d'un service interne de traduction et la gestion d'un cabinet de traduction. Et que je sache, il n'existe guère de cours de formation en gestion qui soient adaptés à ces besoins. Le problème est beaucoup plus aigu dans le cas des traducteurs que dans le cas des professions plus clairement reconnues : médecine, droit, etc., car il n'y a pas de modèle à suivre comme c'est le cas dans ces autres disciplines, tout est à inventer, à tester, à établir. En réalité, il faut gérer l'évolution. Il serait grand temps que les directions des cabinets de traduction se réunissent avec les directions des services internes pour tirer des conclusions à cet effet et partager leurs expériences et leurs préoccupations face à cette fulgurante évolution.

\section{LA CLIENTÈLE}

La clientèle se partage habituellement de la façon suivante :

a) Grandes entreprises avec ou sans service de traduction interne.

b) Petites et moyennes entreprises.

c) Gouvernements et organismes.

d) Clientèle extérieure au Québec ou au Canada.

Si certains cabinets visent un seul type de client, la plupart cherchent sagement d'ailleurs, à diversifier leur clientèle. Pour ma part, je considère qu'une grande entreprise située au Québec et ayant déjà un service interne de traduction, constitue la clientèle idéale pour un cabinet, mais pas nécessairement toujours la plus facile, ni la plus rentable. En effet, nul n'est plus exigeant envers un traducteur qu'un autre traducteur. Mais on arrive toujours à s'adapter aux exigences d'un tel client puisque son objectif professionnel est semblable : une traduction exacte et claire, bref, une bonne communication, bien adaptée au destinataire, une traduction qui sera utile, utilisable et utilisée. L'adaptation aux exigences particulières du client peut prendre trois ou quatre mois, mais elle est possible, et combien intéressante et réconfortante lorsqu'elle est réalisée. Quand un professionnel me dit que la traduction est bonne, je suis sûr qu'elle est excellente. Et je sais que le traducteur qui a la responsabilité du contrôle de la qualité linguistique dans une entreprise s'assurera également de la diffusion des traductions et de la terminologie qu'elles véhiculent.

Lorsqu'il n'y a pas de service interne de traduction, le rôle et les limites du cabinet de traduction doivent être franchement et clairement exposés au client afin de ne pas créer chez lui une expectative non fondée. Et, s'il y a lieu, le cabinet ne doit pas hésiter à conseiller à ce type de client la création d'un service interne, en embauchant d'abord un professionnel chevronné, qui pourra guider l'entreprise dans ses besoins réels et, surtout, contrôler la qualité des services externes, si on décide d'y avoir recours.

La clientèle gouvernementale a des besoins particuliers, et le cabinet de traduction doit prendre une décison selon les mêmes critères qui font que d'autres professionnels 
décident de présenter ou non une offre de services aux gouvernements ou organismes para-gouvernementaux.

Il y a enfin les PME, où il s'agit surtout de s'assurer que l'entreprise en question se doit vraiment de traduire, que le texte n'a pas été traduit ailleurs ou n'est pas sur le point de l'être, que l'on doit accorder la priorité à ce texte en particulier, et enfin, que l'entreprise a les moyens de payer et de rentabiliser le coût de cette traduction.

\section{LE CABINET de TRADUCTION QUÉbÉCOIS ET LA CHARTE DE LA LANGUE FRANÇAISE ( LOI 101 )}

Dans le contexte particulier du Québec, les cabinets de traduction ont le privilège d'avoir un volume de traductions suffisant pour survivre assez bien, comparativement à d'autres cabinets du même type en dehors du Québec ou du Canada. Mais à ce privilège s'accroche nécessairement un devoir, qui est celui de s'assurer que chaque traduction soit nécessaire, qu'elle soit bien faite, qu'elle soit bien utilisée et bien adaptée au public auquel elle est destinée. La tentation semble trop forte pour certains, en effet, de se contenter de tout traduire ce qui passe sans poser de questions. Or, traduire c'est d'abord communiquer, donc servir un but précis. Et dans le contexte québécois en particulier, où la traduction et la recherche terminologique servent à modeler chaque jour une langue du travail, du commerce et des affaires qui soit de plus en plus précise et efficace, et qui serve vraiment à favoriser l'essor de l'économie et non à décorer les tablettes ou à semer la confusion dans un monde où la concurrence est vive et où l'efficacité et la rapidité de l'information et de la communication sont indispensables à la rentabilité et à la survie. Il faut satisfaire aux besoins, bien sûr, mais s'il faut parfois faire vite, il faut aussi faire bien, ce n'est pas nécessairement incompatible, sauf dans le cas de la recherche terminologique, où on doit y mettre le temps voulu, et dans le cas de certaines traductions qui risquent de servir de référence à de multiples fins, tant pour ce qui est du fond que de la forme.

Dans le contexte de l'ensemble socio-linguistique québécois, la diffusion et la qualité de la traduction et de la terminologie ne sont pas aléatoires. Il s'agit d'un élément vital de l'implantation du français comme langue du commerce et des affaires au Québec, et c'est à la seule condition que cet objectif soit atteint, que toute cette masse de traductions acquiert un sens. Autrement, on perpétue une absurdité, soit la traduction massive, sans fin et inutile, sauf pour satisfaire aux exigences d'une loi. En ce sens, un cabinet de traduction conscient de son rôle et de sa véritable utilité rend de précieux services. Dans le cas contraire, il joint les rangs de la masse grouillante, anonyme et sans âme des ramasseurs de piastres et des adeptes de la philosophie du "fast buck".

\section{L'ÉVOLUTION}

Pourquoi le secteur des services (tertiaire disait-on autrefois) est-il encore considéré aujourd'hui, tant par le monde des affaires, du commerce et de l'économie en général que par les instances gouvernementales, comme un secteur qui vit plus ou moins à la remorque, si ce n'est aux dépens des "vrais " moteurs de l'économie, soit les matières premières, la transformation et la fabrication ? Comment se fait-il que personne ne remarque la fragilité, sinon les inquiétants ratés de ces soi-disant " moteurs " de l'économie, et l'augmentation fulgurante de leur asservissement et de leur dépendance face à des secteurs que l'on n'ose plus traiter de services, tant leur force, leur vigueur, leur importance et leur impact s'imposent sur l'ensemble de l'échiquier économique, à savoir l'information et la communication? Aurait-on oublié que la traduction et la terminologie sont des éléments indispensables et indissociables de toute forme d'information et de communication, et surtout en contexte d'informatisation, de télécommunication et de télématique? 
Certains cabinets de traduction recrutent déjà leur clientèle dans plus de la moitié des provinces du Canada et dans trois ou quatre pays. Une recherche terminologique en particulier a entraîné la nécessité d'établir des contacts et des liens dans de nombreux pays. Les réactions ont été spontanément favorables dans chacun de ces pays et à chaque fois, le cabinet en question a dû promettre, en échange d'une documentation généreuse remise à titre gracieux, de faire connaître la date approximative de la publication des résultats de cette recherche terminologique. Nul doute qu'un cabinet ne peut, à lui seul, revendiquer tout le crédit de l'envergure de son travail. Il faut reconnaître la situation particulière des besoins linguistiques du Canada et du Québec. D'autre part, on ne saurait nier l'importance grandissante de l'information et de la communication. Qu'estce qui empêche le Canada, et le Québec en particulier, de devenir, pour ainsi dire, une plaque tournante des communications Amérique-Europe ? Pourquoi ne pas profitr de cette petite avance durement acquise dans le domaine de la traduction et de la recherche terminologique anglais-français et français-anglais ? Notre situation nous a obligés à consacrer beaucoup de ressources humaines et financières à cette fin. Mais si nos besoins sont bien servis, qu'attendons-nous pour faire profiter d'autres clientèles éventuelles de notre expertise et pour rentabiliser un peu tous ces efforts?

Pour un cabinet de traduction et de communication, c'est un objectif lointain, mais non pas inaccessible. Le rêve inaugure et motive la plupart des réalisations... 